\title{
Research on yield forecasting model based on RBF in discrete manufacturing industry application
}

\author{
$\mathrm{Li} \mathrm{Bi}^{1, \mathrm{a}}$, Ruijuan Zhang ${ }^{2, \mathrm{~b}}$ \\ ${ }^{1}$ School of Mathematics and Computer ,Ningxia University, Yinchuan 750021,China \\ ${ }^{2}$ School of Mathematics and Computer ,Ningxia University, Yinchuan 750021,China \\ aemail: billy1968@163.com, bemail: 443153763@qq.com
}

Keywords: Neural Networks; Advanced Planning and Scheduling; prediction model ; Forecast period

\begin{abstract}
Yield forecasting system in discrete manufacturing is a dynamic complex systems with a number of factors, face such a complex and large system is difficult to directly general system to the enterprise, therefore need to give a company's production forecast model. On the background of Wuzhong Instrument company application, this paper by $\mathrm{RBF}$ (radical basis function) neural networks and generalized regression neural network algorithm were compared in enterprise applications, to find the most suitable production forecast model in Wuzhong Instrument company, then Analysis and comparison shows the experimental results. The model has been applied to the valve, spool and valve seat yield prediction in the advanced planning and scheduling in which the practical arrangements played a major role for production planning of Wuzhong Instrument company.
\end{abstract}

\section{Introduction}

Wuzhong Instrument Company is the largest production base of automatic control valve's research and development in china, meanwhile, has developed into the leader of the application of information technology in the same industry throughout the country[1]. With the increase in production, Wuzhong Instrument production plan has become a major restricted factor of expansion and development. In order to increase accuracy of production capacity forecasting, the domestic and foreign scholars have put forward a lot of production forecasting models[2]. but for discrete manufacturing, with small batch production and multiple species' features ,the research of automatic adjustment valve prediction are comparatively far less[3].Therefore, we need to create a set of actual production forecasting model to apply in APS(advanced plan system) of Wuzhong Instrument company.

\section{Description}

In order to meet the established production planning and job scheduling needs, it is necessary for Wuzhong Instrument company which is in the condition of many varieties, small batch-to-order model to solve the problems of batch size and delivery date[4].

With the increasingly severe competition of the market, the Wuzhong Instrument Company begins to pay attention to customers' needs. The production forecasts can predict the peak demand of parts. Then factory can produce some common parts in a relatively low period and reserve them for easing the pressure on the peak of production. Of course, more isn't always better, because warehouse backlog will result in inefficient flow of funds. How to reasonably and accurately predicate production is very important.

\section{Analysis of forecasting model}

Production forecast is that of enterprise according to the existing objective conditions and historical data for the enterprise future development trends and status of the production forecast. 
Enterprises also can surplus in production capacity or a large surplus, the company in accordance with "experience or data support" to determine and "moderate" to arrange some common parts and components to product, in order to avoid wasting manufacturing resources. In addition, post-production also play a relieve pressure[5].

By analyzing the data Wuzhong Instrument factory (2011-2014 year) as well as access to the relevant planning department staff, the indicators Wuzhong Instrument factory production lines were screened to obtain the initial index of production lines.

Wuzhong Instrument obtain from the data analysis, the data of a total of 150,000, but only have the 2000 empty data, part of the field is null, the proportion is very small, so we can take the linear interpolation to fill. Data is based on daily production to record in the database , if the forecast according to daily production volume, the result would be very cumbersome and not intuitive analysis of its regularity, so we follow a month (storage date) such time interval data analysis. There are many product categories, so we conducted an analysis of its valve body, valve sleeve and valve core types.

$$
\overline{x_{i}}=\frac{x_{i}-x_{\min }}{}
$$

Normalization process which can make parameterized in the same range, it can also speed up the training of the network. So before enter the neural network, which is data normalization process, will cover the range of data defined between $[0,1]$.

\section{Research of yield forecasting model}

The yield prediction purpose is to use the program inventory to make no direct relationship between the program and decisive orders, making the production plan have some flexibility and adjustability, so as to solve the problem of inadequate capacity utilization, while reducing to a certain extent production cycle.

Within a production cycle, when the order volume, resulting in production efforts becomes saturated, if the instruction will continue to release a production, the enterprise production capacity is saturated and lead to production cycles. So when production efforts saturated, it should consume inventory, the number of actual production plans should take a minimum. On the contrary, when the orders is little, the enterprise overcapacity, it should to replenish valve parts in orders to use when production capacity is saturated.

$$
T=\left(P_{3}-P_{4}\right)-\left(P_{1}-P_{2}\right)
$$

$\mathrm{T}$ represents minimum inventory than planned over the number of complete or incomplete at year;

$P_{1}$ represents a reasonable production quantities of the product;

$\mathrm{P}_{2}$ represents the actual level of production of the product;

$P_{3}$ represents the product of the month inventory;

$\mathrm{P}_{4}$ represents the smallest year inventory.

$$
P= \begin{cases}0 & T>0 \\ |T| & T \leq 0\end{cases}
$$

$\mathrm{P}$ represents the number of production forecast.

RBF hidden units used as "base" constitutes the hidden layer space, the input vector is mapped directly to the hidden, after space center confirmation determine the mapping is linear [6] between the hidden layer spaces to the output space mapping.

The purpose of RBF neural network output layer and the hidden layer achieved is different, the output layer is linear right adjustments, while the hidden layer of RBF parameters is adjusted, using a nonlinear optimization strategy . 


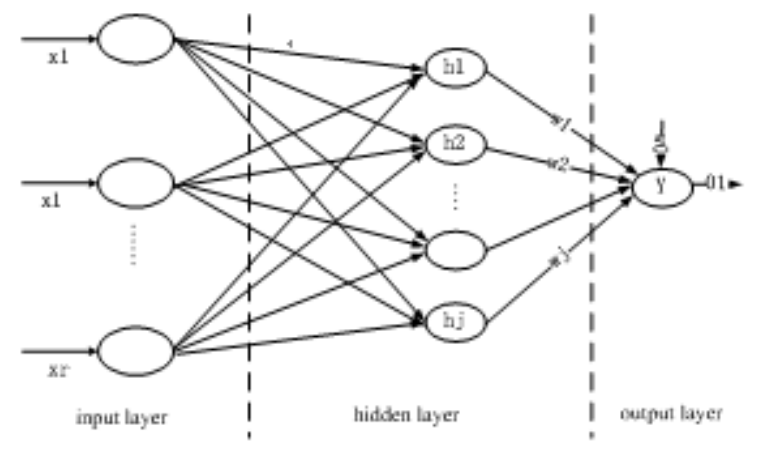

Figure 1 RBF neural network structure

RBF neural network have three layers. The input layer nodes by the signal source; the middle layer is the only network of hidden layers, according to the research object need to set the number of nodes, the selected function called radial basis function; the last layer is the output layer. RBF neural network structure as shown, the distribution function is shown below:

$$
f(x)=w_{0}+\sum_{j=1}^{n} w_{j} h_{j}(x)
$$

$\boldsymbol{n}$ is the number of nodes in the hidden layer neurons, the coefficient $\boldsymbol{W}$ is connection weight; $\boldsymbol{h}$ is the radial basis function.

For RBF neural network, Radial Basis choice is a key issue. The Gaussian function has a simple representation, variables increase does not make the network more complicated and the smoothness are good, the derivative of any order exist, so the general election as radial basis function RBF networks, Gaussian function as shown below:

$$
h(x)=\exp \left(-\frac{(x-u)^{2}}{2 \sigma^{2}}\right)
$$

Another core task is to determine the RBF neural network center, the width and the corresponding connection weights of neurons radial basis functions. In the width of the data reflect the relative position of the respective centers.

In this method of self-organizing learning algorithm chooses RBF center, and the center RBF network $\left(c_{i}\right)$ determine its position to learn through self-organization. Self-organized learning is actually a network resource allocation, the purpose is to learn RBF network located in the center of an important region of the input space, so choose the center to form a specific distribution, which represents the inherent characteristics of the input sample space. After determining a good center, we can determine a good its width.

$$
\begin{aligned}
& \sigma_{i}=\frac{c_{\max }}{\sqrt{2 h}}, i=1,2, \cdots h \text { (6) } \\
& c_{\max } \text { represents the maximum distance between the selected centers. }
\end{aligned}
$$

RBF neural networks often use linear least squares method for solving their weights.

1) Initialization hidden layer to the output layer connection weights $\omega_{i k}$;

2) Give training samples $X^{n}$ and calculate the hidden layer output $R_{i}(x)$;

3) Calculate the output layer output $y_{k}$

4) Calculate the error of the output:

$$
e_{k}=\left|T_{K}-y_{k}\right| \quad k=1,2, \ldots, p
$$

5) Fixed weights:

$$
\omega_{i k}=\omega_{i k}+\eta e_{k} R_{t}(x)
$$

$\boldsymbol{n}$ represents the learning step.

Generalized regression neural network is another form of radial deformation based network. It is based on radial basis function network, it has good performance nonlinear approach, compared with 
the radial basis function network, training more convenient. In Matlab 'newgrnn ()' function can be easily realized by GRNN network.

\section{Case Study}

Since Wuzhong Instrument Factory is based on production orders, demand forecasts based on historical data, it is extremely important, through equation (2) (3) calculated, to obtain the monthly output historical data forecast of 2011-2014 shown in figure 2 for the valve body ,valve sleeve and valve core can be observed in the overall production forecast four years showed a certain regularity, at 6,7,8,12 four months in the valve body production forecast is almost zero, namely valve production efforts tend to be saturated, production efforts in other relatively free month of increase, in order to achieve the goal of balanced productivity, ease valve sleeve, valve body and valve core in peak production efforts.

Our test sample with RBF simulation, first define the sample data, the input vector is defined as a matrix of $5 * 12$, target output value of $1 * 12$ row vector. Divide training data and test data. Using the first January to August training sample derived model, and then the first of September to December, the first sample for testing. The figure5 is displayed RBF neural network during 2013year production forecast to test the actual value and fitting value.

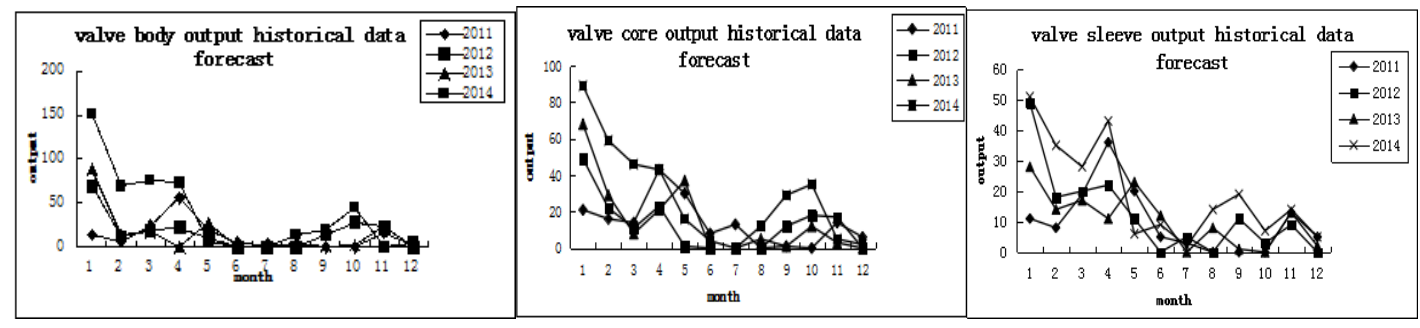

(a) valve body

(b) valve core

(c)valve sleeve

Fig.2. The experimental results

Figure 3 shows, the use of RBF neural network model to predict the yield of the valve body, the valve core and valve sleeve, which can basically reveal their macroscopic yield in three common parts of the law of development. Fitting accuracy is relatively high, the result is acceptable.

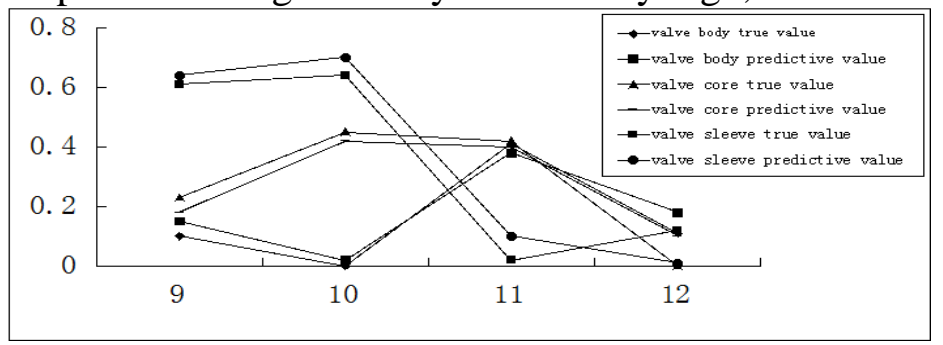

Fig.3. RBF neural network algorithm training result

Finally, respectively using created radial basis function network model and the generalized regression neural network model were tested for September to December samples, and to compare the outputs relative error. The output of two algorithms shows in Table 1 and Table 2 below: 
Table 1 two algorithms output comparison

\begin{tabular}{lcccc}
\hline Part & Month & Actual output value & $\begin{array}{c}\text { RBF neural network } \\
\text { predictive output value }\end{array}$ & $\begin{array}{c}\text { Generalized regression } \\
\text { neural network predictive }\end{array}$ \\
\hline \multirow{2}{*}{ Valvegory body } & 9 & 0.1020 & 0.1550 & 0.2713 \\
& 10 & 0 & 0.0170 & 0.2095 \\
& 11 & 0.4122 & 0.3714 & 0.6667 \\
Valve core & 12 & 0.0991 & 0.1619 & 0.3215 \\
& 11 & 0.6258 & 0.6438 & 0.7454 \\
& 12 & 0.6980 & 0.7912 & 0.9543 \\
Valve sleeve & 11 & 0.0331 & 0.0021 & 0.2339 \\
& 10 & 0.0728 & 0.0854 & 0.1535 \\
& 12 & 0.4357 & 0.0643 & $0 / 1453$ \\
& 10 & 0.4333 & 0.4843 & 0.6575 \\
\hline
\end{tabular}

From table 1,we can see the prediction value RBF neural network predicted an output value are more closer to actual production value than GRNN neural network of enterprises . It can be determined using the RBF neural network is an effective and fitting high precision production forecast methods.

Table 2 two algorithms relative error of comparison

\begin{tabular}{|c|c|c|c|c|c|}
\hline Part Category & & 9 & 10 & 11 & 12 \\
\hline \multicolumn{6}{|l|}{ Month } \\
\hline \multirow[t]{2}{*}{ Valve body } & RBF & 0.0257 & 0.0273 & 0.0171 & 0.0224 \\
\hline & GRRN & 0.1834 & 0.3754 & 0.2854 & 0.3327 \\
\hline \multirow[t]{2}{*}{ Valve core } & RBF & 0.0363 & 0.0375 & 0.0323 & 0.0113 \\
\hline & GRRN & 0.2544 & 0.1735 & 0.3563 & 0.4621 \\
\hline \multirow[t]{2}{*}{ Valve sleeve } & RBF & 0.0232 & 0.1112 & 0.0534 & 0.0173 \\
\hline & GRRN & 0.3511 & 0.4214 & 0.3221 & 0.3321 \\
\hline
\end{tabular}

From table2 we can found that using RBF neural network model yields prediction error are relatively less than GRRN neural network model, so we can determine use RBF neural network model to forecast production of valve body, valve core and valve sleeve.

\section{Conclusion}

We mainly used RBF neural network training forecast data on yield, to compare with the results generalized neural network training, we found that the result of RBF neural network simulation are more closer to the actual value of the enterprise production forecast and error with respect to the generalized regression neural network small enough, so we will use RBF neural network training model in Wuzhong Instrument APS system, to achieve a reasonable arrangement some valve parts of the production plan. It can avoid the waste of manufacturing resources and also relieve pressure on production peak production role. The next step we need to find out the dependencies, resource constraints relation between the valve parts further amended effective predictive models. 


\section{Acknowledgement}

In this paper, the research was sponsored by the National Science and Technology Support Program (Project No. 2013BAF02B05) and the National Emergency Management Project (Project No. 61440046).

\section{References:}

[1]MaYushan, Pan ERP-integration of the two road to success [M].Beijing: Mechanical Press, 2012.3.

[2]Weihai Kun theory and neural network architecture design [M]. Beijing: National Defense Industry Press, 2005

[3] Liu Liang. Research demand management base on APS[D].Beijing Jiaotong University.2010.

[4]Zhou lu. Analysis of supply chain inventory management[J].Industrial design.2011(5)

[5]Xichong Bing. Demand forecasting and inventory management[J].Logistics technology and application.2011(3) 